\title{
Gaussian Mixture Embedding of Multiple Node Roles in Networks
}

\author{
Yujun Chen • Juhua Pu • Xingwu Liu • \\ Xiangliang Zhang
}

Received: date / Accepted: date

\begin{abstract}
Network embedding is a classical topic in network analysis. Current network embedding methods mostly focus on deterministic embedding, which maps each node as a low-dimensional vector. Thus, the network uncertainty and the possible multiple roles of nodes cannot be well expressed.

In this paper, we propose to embed a single node as a mixture of Gaussian distribution in a low-dimensional space. Each Gaussian component corresponds to a latent role that the node plays. The proposed approach thus can characterize network nodes in a comprehensive representation, especially bridging nodes, which are relevant to different communities. Experiments on real-world network benchmarks demonstrate the effectiveness of our approach, outperforming the state-of-the-art network embedding methods. Also, we demonstrate that the number of components learned for each node is highly related to its topology features, such as node degree, centrality and clustering coefficient.
\end{abstract}

\footnotetext{
Yujun Chen

Beihang University

King Abdulah University of Science and Technology

E-mail: chenjohn@buaa.edu.cn

Juhua $\mathrm{Pu}$

Beihang University

Research Institute of Beihang University in ShenZhen

E-mail: pujh@buaa.edu.cn

Xingwu Liu

Institute of Computating Technology, Chinese Academy of Sciences

E-mail: xingwu.liu@ict.ac.cn
}

Xiangliang Zhang®

King Abudulah University of Science and Technology

E-mail: xiangliang.zhang@kaust.edu.sa 


\section{Introduction}

Network embedding, which maps nodes in a low-dimension space, has been proven successful for benefiting a variety of downstream network mining tasks, such as node classification, community detection, link prediction and visualization in citation networks (e.g., arXiv, Aminer), gene interactions (e.g., Protein-Protein interactions), social or commercial networks (e.g., Twitter, Facebook) [1-4]. The core idea behind a number of network embedding methods is to leverage the high-dimensional graph structure information, such as graph adjacency or graphlet, to a dense vector representation, i.e., a single point in low-dimensional space $[2,3,5,7]$. Embedding vectors of nodes are similar if they are geographically close in the original graph. Such deterministic embedding vectors have limitations on expressing the different social roles comprehensively nodes are playing with uncertainty.

In real networks, the uncertainty of network representation is typically present on nodes that play multiple roles in different communities. For example, a Wikipedia page about Kobe Bryant, a famous athlete and businessman, can be labeled as sports, celebrities, marketing and entertainment, etc. Also, in social media websites, users often join different social groups, such as sports groups, digital groups, and machine learning study groups simultaneously. These versatile users or pages belong to diverse communities. Embedding them as a single point vector cannot naturally express such multiformity of a node. Bojchevski and Günnemann [8] recently proposed an interesting and inspiring approach, embedding each node by a Gaussian distribution to address the uncertainty. Specifically, they learn the mean and variance of embedding by a dense non-linear neural network. However, the obtained single Gaussian distribution embedding cannot characterize the multiple roles that a node can play, and then will still affect downstream network analysis tasks.

In this paper, we propose to characterize the uncertainty of node embedding by a mixture of Gaussian. Fig. 1 demonstrates a motivation example of our proposed approach. Suppose there is a network in Fig. 1a presenting the club members relations. Nodes in the same club are colored in the same color, while those belonging to both groups are colored by green. Fig. 1(b) shows the deterministic embedding of nodes obtained by Deepwalk [7], while Fig.1(c) is the mixture Gaussian embedding produced by our methods. It can be seen that our embedding results can better capture the variance caused by multi-group nodes. For example, bridging node 1 is not mapped to the middle position as in Fig.1 (b), but has a learned representation with two Gaussian components, 1(0) and 1(1) in Fig.1(c), same for node 0 and 2.

Our proposed method called Node2Mixture learns for each node a mixture of Gaussian distribution as a new representation. The objective function is defined based on the expected likelihood kernel [9], which measures the similarity of two mixture distributions. By maximizing the objective function, Node2Mixture not only finds out how many different roles a node is playing, also estimates the uncertainty related to the roles. 


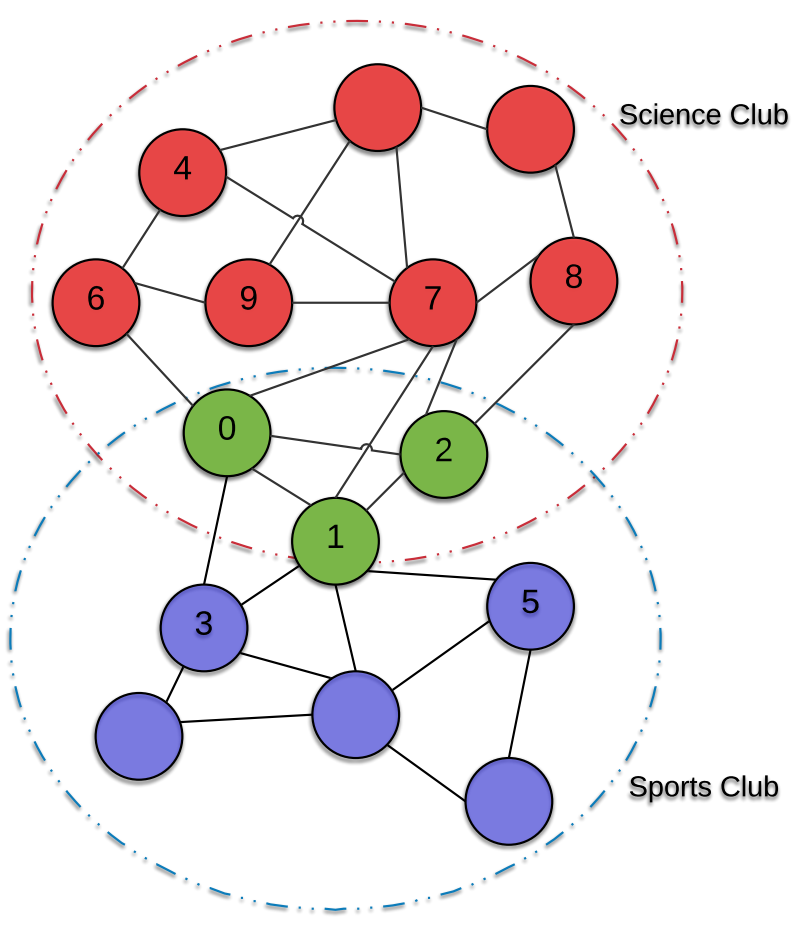

(a) Input: Plain Graph

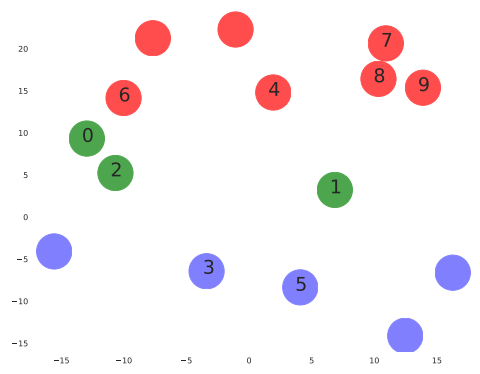

(b) Deterministic Embedding by Deepwalk

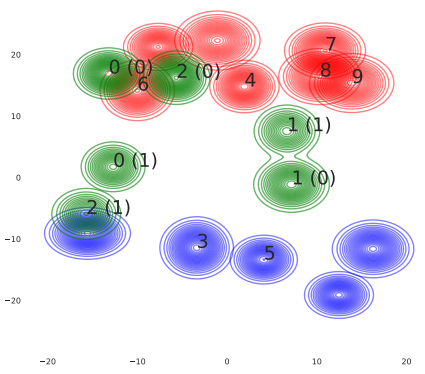

Fig. 1: Our proposed method learns a mixture distribution for each node in a plain network. Node color indicates the community label, while nodes belonging to both groups are colored by green. Nodes used in (a) are plotted in (c) with mixture component ID, e.g., 1(0) and 1(1) are the representation of node 1 with two Gaussian components. Bridging nodes in green are mapped to representations with the mixture of more components than other nodes. 
We test the obtained representation on the commonly used graph classification task in three benchmark networks: Citeseer, PPI and Wikipedia. We contrast the performance of Node2Mixture with the state-of-the-art network embedding learning methods, such as VERSE [5], Graph2Gauss [8], Node2Vec [2], DeepWalk [7] and LINE [3]. The empirical experimental results show that using mixture of Gaussians can better model nodes with multiple roles. We also analyze the relevance of the number of Gaussian components in learned embedding to network features, such as node centrality, and clustering coefficient. Our findings confirm that Node2Mixture can obtain more meaningful embeddings for network analysis.

Our contributions in this work can be summarized as follow:

- We propose Node2Mixture to represent network nodes as the expressive Gaussian mixture distribution that explicitly learns the multiple characteristics of nodes.

- The proposed Node2Mixture is efficient with computational complexity linear to graph size, making it applicable to arbitrary types of networks, and scalable to millions of nodes.

- We show how Node2Mixture models the uncertainty of node representations by network features.

- We empirically evaluate Node2Mixture for several network learning tasks, and show that Node2Mixture outperforms the state-of-the-art approaches.

\section{Related work}

\subsection{Network Embedding}

Network embedding is an active research area in recent years, with a number of developed learning methods. Recent review papers provide comprehensive introduction to the existing methods $[4,10,11]$. Here we focus on discussing only the unsupervised embedding methods at plain graphs (using only network topology), referring the other semi-supervised and attributed network embedding methods to $[4,10-14]$. According to the relevance to our problem, these methods fall into two categories: learning node representation as vectors or as distributions.

\subsubsection{Network Embedding as Vectors}

Currently, the majority of network embedding methods are in this category. Given the input of a plain network $G=(V, E)$, the problem is to represent each node $v \in V$ as a $d$-dimensional vector, where $d(d \ll|V|)$ is a predefined dimensionality parameter. Previous methods in network embedding include spectral techniques $[1,16]$ and non-linear dimensionality reduction [17]. Such methods are not capable of applying to large graphs, as they operate on dense matrices. Meanwhile, advances in machine learning made techniques like using 
neural methods available for learning representations [18]. Inspired by the success of natural language processing (NLP) methods in representation learning, many recent methods transfer word embedding approaches to the network embedding area [2,7,20,21]. Approaches like Deepwalk [7] and Node2Vec [2] focus on plain graphs, by exploiting neighborhood nodes with short random walk and then using the Skip-gram model [22]. Also, Struct2Vec [23] captures the isomorphism among nodes and structural similarities, and builds the representation based on Deepwalk core. Other work introduces higher-order proximity between nodes to the embedding process, such as LINE [3] and, proNE [63] and VERSE [5]. More recently, using matrix factorization to generate proximity matrix is a new thread. Papers like [64-66] optimize the representation by incorporating the proximity matrix.

All these approaches produce deterministic embedding vectors for nodes, without considering the uncertainty. Recent papers [24, 26-28,62] study the possibility of generating multiple representations for nodes. For example, [28] proposes a multi-sense embedding method for heterogeneous network. Each node can have various representations depending on the heterogeneous neighborhood nodes. [26] organizes node embeddings in a hierarchical setting for heterogeneous network, while [27] studies the same hierarchical network embedding problem in homogeneous network scenario. [62] proposed a method to learn multiple representations for each node by incorporating community structures.

\subsubsection{Network Embedding as Distribution}

A few approaches solve the uncertainty problem of node embedding by learning a distribution. For example, He et al. [29] represent knowledge graph nodes as a single Gaussian distribution, with both $d$-dimensional mean and $d$-dimensional variance. Dos Santos et al. [30] study to learn Gaussian distribution representation for node classification in heterogeneous graphs. Here our studied problem is the unsupervised plain network embedding, where both approaches are not applicable. The most relevant work is [8], which uses a Gaussian distribution to model a node (Graph2Gauss). Further, [67] follows the idea and proposes another VAE approach for graph data by representing each node as a Gaussian distribution with Wasserstein distance. [68] also learns the Gaussian Embeddings for users and used for recommendation tasks. This approach is capable of capturing the uncertainty in complex networks, and better deals with link prediction problem using extra information, such as variance. However, in other problems like node classification or community detection, Graph2Gauss still relies on the mean vector [8], which is almost the same as using a single point embedding vector. Also, the usage of a single Gaussian distribution for one node limits the characterization of multiple roles a node can play. 


\subsection{Gaussian Mixture and Representation Learning}

Gaussian Mixture model (GMM) [31] is a parametric probability model representing data generating function as a weighted sum of several Gaussian components. GMM has been commonly used for various learning tasks, including classification [32], image segmentation [32], speech recognition [33,35] and data clustering [36]. Meanwhile, there has been a growing interest in joint learning of dimensionality reduction (feature learning) and Gaussian mixture modeling. For example, Yang et al. [37] propose to jointly learn linear dimensionality reduction. Paulik et al. [38] use mixture model for pre-training the feature selection. Also, Gaussian mixture models are used to learn the representation of words $[39,40]$. Unlike the existing work of using Gaussian mixture distribution, our method focuses on learning node representations through preserving the node geometric relations in network topology.

\subsection{Energy Based Learning}

Our objective function setting and optimization are relevant to energy-based learning, which has been actively used for unsupervised representation learning [41], natural language processing [42], text embedding [43] and computer vision $[44,46]$. The essence of energy based learning is to build an energy function $E_{\theta}(x, y)$ that can learn the parameter $\theta$ based on pairs of inputs $x$ and $y$. In unsupervised setting, $x$ and $y$ are always served as the score of positive and negative input energy, respectively. The energy function is often served as the objective function and combined with sophisticated learning architectures, such as auto-encoder, deep belief networks, or generative adversarial networks. In network embedding, energy based learning is also used in [8]. The parameter $\theta$ is regarded as the learned node representation [8], and $x, y$ are the positive and negative energy score for pairs of nodes. Our work builds the objective function inspired by the energy based learning methods in [41], using the hinge energy loss with $L 2$ norm:

$$
L_{\theta}\left(Y^{i}, X^{i}\right)=\max \left(0, m+E_{p o s}-E_{n e g}\right)+\lambda\|\theta\|_{2}^{2} .
$$

More details are given next.

\section{Methodology}

In this section, we describe the Node2Mixture model and discuss in detail on how to address the learning problem.

\subsection{The Mixture Gaussian Representation of nodes}

Let $G=(\mathbf{V}, \mathbf{E})$ be an undirected graph, where $\mathbf{V}$ denotes the set of $N$ nodes and $\mathbf{E} \subseteq \mathbf{V} \times \mathbf{V}$ is the set of edges. For the $i$-th node $v_{i}$, we aim to represent 
it as a mixture of Gaussian distribution with $K$ components

$$
\begin{aligned}
p_{i}(\mathbf{x}) & =\sum_{k=1}^{K} \pi_{k} \mathcal{N}\left(\mathbf{x} \mid \boldsymbol{\mu}_{i, k}, \boldsymbol{\Sigma}_{i, k}\right) \\
& =\sum_{k=1}^{K} \pi_{k} \frac{1}{\sqrt{(2 \pi)^{d} *\left|\boldsymbol{\Sigma}_{i, k}\right|}} e^{-\frac{1}{2}\left(\mathbf{x}-\boldsymbol{\mu}_{i, k}\right)^{T} \boldsymbol{\Sigma}_{i, k}^{-1}\left(\mathbf{x}-\boldsymbol{\mu}_{i, k}\right)},
\end{aligned}
$$

where $\pi_{k}$ is the mixing coefficients with $\sum_{k=1}^{K} \pi_{k}=1$. The mean vector $\boldsymbol{\mu}_{i, k}$ is the location of the $k$-th Gaussian component in the representation space $\mathbb{R}^{d}$, $d \ll N$. And $\boldsymbol{\Sigma}_{i, k} \in \mathbb{R}^{d \times d}$ is the corresponding covariance matrix of the $k$-th Gaussian component. Intuitively, each Gaussian component characterizes one type of the role a node plays in one community.

\subsection{The Symmetric Similarity Measure}

To preserve the node geometric relations in network topology, neighboring nodes $n_{i}$ and $n_{c}$ are expected to have similar embeddings $p_{i}$ and $p_{c}$, given in Eq. (3). Recall that most skip-gram based network embedding models measure the similarity of two embedding vectors by dot product [2,7]. However, dot product is not valid for comparing two distributions in our case. A straightforward way to measure distribution similarity is to use the $\mathrm{KL}$ divergence, which is also used in Graph2Gauss [8]. However, the KL divergence does not have a closed form for two Gaussian mixture distributions [47].

The probability product kernel [9] is more suitable for the similarity measurement of Gaussian mixtures. It provides a general inner product, which is evaluated as the integral of the product of pairs of distributions. In this paper, according to [48], the special case of probability product kernel, expected likelihood kernel is employed for comparing the mixture distributions.

Expected likelihood kernel takes the form of the expectation of one distribution under the other. For two nodes with mixture distributions $p_{i}(\mathbf{x})=$ $\sum_{k_{i}} \pi_{k_{i}} \mathcal{N}\left(\mathbf{x} \mid \boldsymbol{\mu}_{i, k_{i}}, \boldsymbol{\Sigma}_{i, k_{i}}\right)$ and $p_{c}(\mathbf{x})=\sum_{k_{c}} \pi_{k_{c}} \mathcal{N}\left(\mathbf{x} \mid \boldsymbol{\mu}_{c, k_{c}}, \boldsymbol{\Sigma}_{c, k_{c}}\right)$, their similarity is then defined as

$$
E\left(p_{i}, p_{c}\right)=\int_{x \in \mathbb{R}^{d}} p_{i}(\mathbf{x}) p_{c}(\mathbf{x}) d \mathbf{x}
$$


which can be reduced to the sum of $k_{i} \times k_{c}$ elementary expected likelihood sub-kernels after simple calculus derivations:

$$
\begin{aligned}
E & \left(p_{i}, p_{c}\right) \\
& =\int_{\mathbf{x} \in \mathbb{R}^{d}}\left(\sum \pi_{i} \mathcal{N}\left(\mathbf{x} \mid \boldsymbol{\mu}_{i, k_{i}}, \boldsymbol{\Sigma}_{i, k_{i}}\right)\right) \times\left(\sum \pi_{i} \mathcal{N}\left(\mathbf{x} \mid \boldsymbol{\mu}_{i, k_{i}}, \boldsymbol{\Sigma}_{i, k_{i}}\right)\right) d \mathbf{x} \\
& =\sum_{\pi_{k_{i}}} \sum_{\pi_{k_{c}}} \pi_{k_{i}} \pi_{k_{c}} \int_{\mathbf{x} \in \mathbb{R}^{d}} \mathcal{N}\left(\mathbf{x} \mid \boldsymbol{\mu}_{i, k_{i}}, \boldsymbol{\Sigma}_{i, k_{i}}\right) \times \mathcal{N}\left(\mathbf{x} \mid \boldsymbol{\mu}_{c, k_{c}}, \boldsymbol{\Sigma}_{c, k_{c}}\right) d \mathbf{x} \\
& =\sum_{\pi_{k_{i}}} \sum_{\pi_{k_{c}}} \pi_{k_{i}} \pi_{k_{c}} \mathcal{N}\left(0 \mid \boldsymbol{\mu}_{i, k_{i}}-\boldsymbol{\mu}_{c, k_{c}}, \boldsymbol{\Sigma}_{i, k_{i}}+\boldsymbol{\Sigma}_{c, k_{c}}\right)
\end{aligned}
$$

It can be seen that the expected likelihood kernel can capture the similarity between pairs of hidden representations. Eq. (5) sums all the possible pairs of similarities together with weighted average by mixture coefficient $\pi_{k, i}$ and $\pi_{k, c}$. However, using expected likelihood directly in training will be numerically hard, as the quantities can get exponentially small and difficult to deal with. In result, we choose to use the logarithm of $E\left(p_{i}, p_{c}\right)$ as the energy function. According to Eq. (5), the log energy (in $d$ dimension) is

$$
\begin{aligned}
& \log E\left(p_{i}, p_{c}\right) \\
& \quad=\log \sum_{\pi_{k_{i}}} \sum_{\pi_{k_{c}}} \pi_{k_{i}} \pi_{k_{c}} \mathcal{N}\left(0 \mid \boldsymbol{\mu}_{i, k_{i}}-\boldsymbol{\mu}_{c, k_{c}}, \boldsymbol{\Sigma}_{i, k_{i}}+\boldsymbol{\Sigma}_{c, k_{c}}\right) \\
& \quad=\log \sum_{\pi_{k_{i}}} \sum_{\pi_{k_{c}}} \pi_{k_{i}} \pi_{k_{c}} \sigma_{k_{i}, k_{c}}
\end{aligned}
$$

where

$$
\begin{aligned}
& \sigma_{k_{i}, k_{c}}=\exp \left[-\frac{1}{2} \log \operatorname{det}\left(\boldsymbol{\Sigma}_{i, k_{i}}+\boldsymbol{\Sigma}_{c, k_{c}}\right)-\frac{d}{2} \log (2 \pi)\right. \\
& \left.-\frac{1}{2}\left(\boldsymbol{\mu}_{i, k_{i}}-\boldsymbol{\mu}_{c, k_{c}}\right)^{T}\left(\boldsymbol{\Sigma}_{i, k_{i}}+\boldsymbol{\Sigma}_{c, k_{c}}\right)^{-1}\left(\boldsymbol{\mu}_{i, k_{i}}-\boldsymbol{\mu}_{c, k_{c}}\right)\right]
\end{aligned}
$$

The $\sigma_{k_{i}, k_{c}}$ is the $\log$ form of $\mathcal{N}\left(0 \mid \boldsymbol{\mu}_{i, k_{i}}-\boldsymbol{\mu}_{c, k_{c}}, \boldsymbol{\Sigma}_{i, k_{i}}+\boldsymbol{\Sigma}_{c, k_{c}}\right)$. Turning the normal distribution as the exponential form can make the gradient easier numerically. It is trivial to obtain the gradient of the energy function with respect to the means $\boldsymbol{\mu}_{i}, \boldsymbol{\mu}_{c}$ and covariance $\boldsymbol{\Sigma}_{i}, \boldsymbol{\Sigma}_{c}$.

\subsection{The Energy-based Loss}

Given the pairwise constraint defined in Eq. (5), it is always intractable to optimize the function directly by the positive samples [49]. We use an energy based loss function to address this issue. By employing the hinge loss [41], which is intensively used in unsupervised learning approaches [50], our objective function is defined as:

$$
\mathcal{L}=\sum_{n_{i} \in V} \frac{1}{Z} \sum_{n_{c} \in D_{i}} \sum_{n_{\bar{c}} \in D_{i}^{\prime}} \mathcal{M}\left(i, n_{c}, n_{\bar{c}}\right)+\frac{1}{2} \lambda\|\theta\|_{2}^{2},
$$


where

$$
\mathcal{M}\left(i, n_{c}, n_{\bar{c}}\right)=\max \left(0, m+\log E\left(p_{i}, p_{c}\right)-\log E\left(p_{i}, p_{\bar{c}}\right)\right)
$$

where $D_{i}$ is the positive node set for node $n_{i}$, and $D_{i}^{\prime}$ is the negative node set for $n_{i} . \theta$ is the parameters set for energy function $\log E\left(p_{i}, p_{c}\right)$, such as $\boldsymbol{\mu}, \pi$, and $\boldsymbol{\Sigma} . Z=\sum_{i \in V}\left|D_{i}\right|+\left|D_{i}^{\prime}\right|$ is a normalization term. Also, we add an $L_{2}$ regularization term for all parameters, weighted by a hyper-parameter $\lambda$. The difference between the positive node pair energy and the negative node pair energy is penalized linearly when larger than $-m$.

Following the idea of skip-gram model, neighborhood nodes with shorter geodesic distance should be closer in the embedding space, while the nodes further apart in the network should be penalized if the energy is small [7]. We adopt the second-order random walk graph sampling method [2] to form positive and negative sets. In brief, a node sequence $T=\left\{n_{1}, n_{2}, \ldots, n_{|T|}\right\}$ can be generated by the second-order random walk. Nodes $n_{c} \in\left\{n_{i-w}, \ldots, n_{i+w}\right\} \backslash n_{i}$ are regarded as the context nodes of the center node $n_{i}$, i.e., positive nodes, where $w$ is the window size. Negative nodes are obtained by negative sampling $n_{\bar{c}} \in\left\{\mathbf{V} \backslash\left\{n_{i-w}, \ldots, n_{i+w}\right\}\right\}$. For each node $n_{i}$, we restrict the number of positive nodes $n_{c}$ is the same as number of negative nodes $n_{\bar{c}}$.

The employment of hinge loss has two advantages. First, there is an absolute margin to separate positive and negative node pairs rather than a relative relation as skip-gram. Second, the hinge loss only depends on the energy differences, and does not constrain individual energies to any specific value. The expected likelihood kernel energy is not a bounded function, hence the hinge loss is well suited for hinge loss. Other commonly used loss functions like square-square loss [51] or square-exponential loss [8] can also be alternatively used. However, they all need a value constraint for positive or negative energy, which will be difficult to estimate.

\subsection{Relation to Other Models}

Node2Mixture is related to both skip-gram based methods [2,7] and distribution based model [8]. We discuss their relevance next.

\subsubsection{Skip-gram Based Methods}

Both Deepwalk [7] and Node2Vec [2] aim to maximize the probability of the co-occurrence of node $n_{i} \in V$ and its context node $n_{c} \in V$, which can be formalized as:

$$
\max \prod_{n_{i} \in V} \prod_{n_{c} \in N_{S}\left(n_{i}\right)} \operatorname{Pr}\left(n_{c} \mid n_{i}\right)
$$

where $N_{S}\left(n_{i}\right)$ is the network neighborhood for node $n_{i}$. For most skip-gram based models, this relationship is bounded by binary entropy loss function 
with energy function $E(i, c)$. The energy function is formalized as dot product of vectors:

$$
E(i, c) \propto \boldsymbol{e}_{i}^{T} \boldsymbol{e}_{c},
$$

where $\boldsymbol{e}_{i}$ and $\boldsymbol{e}_{c}$ are embeddings for node $n_{i}$ and $n_{c}$.

For our mixture Gaussian model, if we select the mixture number $K=1$, and fix the covariance for all nodes as $\boldsymbol{\Sigma}_{i}=\boldsymbol{\Sigma}_{c}=\frac{1}{2} \mathbf{I}$, and $\boldsymbol{\mu}_{i}=\boldsymbol{e}_{i}, \boldsymbol{\mu}_{c}=\boldsymbol{e}_{c}$, then the expected likelihood kernel energy can be re-written as:

$$
\begin{aligned}
\log E\left(p_{i}, p_{c}\right) & =\log \mathcal{N}\left(0 \mid \boldsymbol{\mu}_{i}-\boldsymbol{\mu}_{c}, \boldsymbol{\Sigma}_{i}+\boldsymbol{\Sigma}_{c}\right) \\
& =\log \mathcal{N}\left(0 \mid \boldsymbol{e}_{i}-\boldsymbol{e}_{c}, \mathbf{I}\right) \\
& \propto\left(\boldsymbol{e}_{i}-\boldsymbol{e}_{c}\right)^{T}\left(\boldsymbol{e}_{i}-\boldsymbol{e}_{c}\right) \\
& =\left\|\boldsymbol{e}_{i}\right\|+\left\|\boldsymbol{e}_{c}\right\|-2 \boldsymbol{e}_{i}^{T} \boldsymbol{e}_{c} .
\end{aligned}
$$

Recall that skip-gram model can be normalized to unit length for better similarity calculation [52], $\left\|\boldsymbol{e}_{i}\right\|$ and $\left\|\boldsymbol{e}_{c}\right\|$ can be regarded as constants, and the upper energy function is only related to $\boldsymbol{e}_{i}^{T} \boldsymbol{e}_{c}$ as the form:

$$
\begin{aligned}
\log E\left(p_{i}, p_{c}\right) & =C-2 * \boldsymbol{e}_{i}^{T} \boldsymbol{e}_{c} \\
& \propto \boldsymbol{e}_{i}^{T} \boldsymbol{e}_{c}
\end{aligned}
$$

which is the same as Eq. (12).

\subsubsection{Distribution based Models}

The recently published method Graph2Gauss [8] naturally extends single vector representation to a Gaussian distribution, for capturing the uncertainty of node embedding vectors. If setting the mixture number $K=1$, our Node2Mixture and Graph2Gauss both embed each node as a single Gaussian distribution. Another important difference is that Graph2Gauss uses the KL-divergence as the energy between two Gaussian distributions, while we use the Probability Product Kernel. The KL-divergence and Probability Product Kernel are two related probabilistic kernels. Specifically, the KL-divergence is relate to a special case of Probability Product Kernel, the Bhattacharyya Kernel [9]. However, in the cases that $K>1$, probability Product Kernel takes the advantage that it can naturally deal with the inner product of Gaussian mixture model, while KL-divergence has no closed form expression, it can only be approximated [53].

\subsection{Discussion}

\subsubsection{Training}

The objective function Eq. (9) is differentiable due to the fact that logarithm function is a smooth function. The gradient of the energy function with respect 
to the means $\boldsymbol{\mu}$ and covariances $\boldsymbol{\Sigma}$ is:

$$
\begin{gathered}
\frac{\partial \log E\left(p_{i}, p_{j}\right)}{\partial \boldsymbol{\mu}_{i, k_{i}}}=-\frac{\partial \log E\left(p_{i}, p_{j}\right)}{\partial \boldsymbol{\mu}_{j, k_{j}}}=-\Delta_{i, j, k_{i}, k_{j}} \\
\frac{\partial \log E\left(p_{i}, p_{j}\right)}{\partial \boldsymbol{\Sigma}_{i, k_{i}}}=-\frac{\partial \log E\left(p_{i}, p_{j}\right)}{\partial \boldsymbol{\Sigma}_{j, k_{j}}} \\
=\frac{1}{2}\left(\Delta_{i, j, k_{i}, k_{j}} \Delta_{i, j, k_{i}, k_{j}}^{T}-\left(\boldsymbol{\Sigma}_{i, k_{i}}+\boldsymbol{\Sigma}_{j, k_{j}}\right)^{-1}\right)
\end{gathered}
$$

where

$$
\Delta_{i, j, k_{i}, k_{j}}=\left(\boldsymbol{\Sigma}_{i, k_{i}}+\boldsymbol{\Sigma}_{j, k_{j}}\right)^{-1}\left(\boldsymbol{\mu}_{i, k_{i}}+\boldsymbol{\mu}_{j, k_{j}}\right)
$$

The hinge loss term in Eq. (9) is not differentiable, and thus causes difficulties to use gradient descent directly. However, the hinge loss has a sub-gradient with respect to the parameters $\boldsymbol{\mu}$ of the Gaussian mixture with the energy difference is given by:

$$
\begin{gathered}
\frac{\partial \mathcal{L}}{\partial \boldsymbol{\mu}}=\sum_{n_{i} \in V} \frac{1}{Z} \sum_{n_{c} \in D_{i}} \sum_{n_{\bar{c}} \in D_{i}^{\prime}} \frac{\partial \mathcal{M}}{\partial \boldsymbol{\mu}}+\lambda\|\boldsymbol{\mu}\| \\
\frac{\partial \mathcal{M}}{\partial \boldsymbol{\mu}}=\left\{\begin{array}{l}
\frac{\partial \log E\left(p_{i}, p_{\bar{c}}\right)}{\partial \theta}-\frac{\partial \log E\left(p_{i}, p_{c}\right)}{\partial \theta} \\
\text { if } m<\log E\left(p_{i}, p_{c}\right)-\log E\left(p_{i}, p_{\bar{c}}\right) \\
\text { if } m>\log E\left(p_{i}, p_{c}\right)-\log E\left(p_{i}, p_{\bar{c}}\right)
\end{array}\right.
\end{gathered}
$$

In the experiments, both diagonal and spherical covariance matrices are used with hard eigenvalues constraint to make efficient computations, and the $\sigma_{k_{i}, k_{c}}$ in Eq. (7) will be easier to compute. Also, controlling the bottom eigenvalues of the covariance is especially important when training with expected likelihood, since the energy function includes a log det term that can give very high scores to small covariances, dominating the rest of the energy. The covariance matrices need to be kept positive definite as well as reasonably sized [51].

\subsubsection{Selecting the Number of Mixtures}

Many recent approaches determine the hyper-parameter of mixture number after trial and errors. However, such process is neither efficient nor necessary. It is possible to infer the possible number of mixtures before the training phase. Here we adopt two different model selection methods, Bayesian Information Criterion and Dynamic mixture embedding learning.

Bayesian Information Criterion: Bayesian Information criterion for high dimensionality [54] is always used for model selection problems. The Bayesian information criterion is formally defined as:

$$
B I C=-2 \log \left(\sum \log E\left(p_{i}, p_{c}\right)\right)+\gamma \log \left(p_{n}\right) k,
$$


where $p_{n}$ denotes the number of training edges, $\gamma$ is a multiplicative factor and is 1 by default, and $k$ is the number of parameters be estimated, $2|V| d$ here. The first term $\sum \log E\left(p_{i}, p_{c}\right)$ denotes the likelihood function of our model, which reflects the goodness-of-fit for our data. The log likelihood function is calculated as the sum of all training edges' value of expected likelihood kernel. The second term is the penalty for the Gaussian model's parameter complexity. The Bayesian Information Criterion is data dependent, because the likelihood and number of parameters are related to data. We present empirical results in the experimental section (Section 4.2) for the model section using BIC.

Dynamic Mixture Estimation: It is nice to determine the number of mixtures before training. However, it is still not plausible that all nodes should be with the same number of mixtures. To capture the dynamically increasing numbers of Gaussian for each node, we adopted a simple dynamic mixture embedding learning approach. We initialize the model by setting all nodes' mixture coefficient to be a one-hot vector, which indicates that all nodes should only have one role component at the beginning of the training. During the training, the mixture coefficient is automatically updated. Following the aforementioned updating process, an additional non-linear mask, rectified linear unit (ReLU), is used for mixture coefficient updating for each node. Combining the ReLU operator, the gradient for mixture coefficient $\pi_{i, k_{i}}$ is:

$$
\frac{\partial L}{\partial \pi_{i, k_{i}}}=\delta\left(\sum_{\pi_{k_{c}}} \pi_{k_{c}} \sigma_{k_{i}, k_{c}}\right) .
$$

where $\delta(\cdot)$ denotes the rectified linear unit (ReLU) activation function applied to the coefficient updating. By using ReLU activation, we assume all the learned network weights with negative values are not activated. For node $n_{i}$, only if the context distribution is much closer to the zero weight distribution that makes the gradient of zero weight positive, the zero weight Gaussian distribution will be activated as one component of the mixture Gaussian. After training, the mixture of Gaussian will be the combination of Gaussian with positive weights.

\subsubsection{Computational Complexity}

Node2Mixture is highly efficient. The time complexity for computing our loss is $O(|V|)$, where $|V|$ denotes the number of nodes. First, we adopt the Node2Vec sampling method with $O(|V|)$ complexity. In the training phase, we use minibatch gradient descent to learn the variables. The computation of each pair of nodes is fully independent and can easily be parallelized, so the training phase can be considered as constant computational complexity. As a result, the total complexity is $O(|V|)$, which is linear to the number of nodes. 


\section{Experiments}

We have performed comparative evaluation of Node2Mixture models against a wide variety of strong baselines and previous approaches, on graph learning tasks, achieving or matching the state-of-the-art performance across all of them. This section summarizes our experimental setup, results, and also analyzes Node2Mixture model's representations.

\subsection{Experiment Setups}

\subsubsection{Datasets}

We utilize both multi-class and multi-label benchmark network datasets in our experiments. To fit our methods, we use three topology only benchmark datasets:

- Citeseer [55]: This is a multi-class citation network dataset about citation relations in Citeseer website. The network has 3,312 nodes and 4,732 links, and 6 labels for nodes.

- Protein-Protein Interaction network (PPI) [56]: This is a multi-label biological network dataset. The graph is a subgraph of the PPI network for Homo Sapience. The network has 3,890 nodes, 76, 584 edges, and 50 different labels.

- Wikipedia [58]: This is an multi-label word network dataset. The graph is a co-occurrence network of words appearing in the first million bytes of Wikipedia dump. The network has 4,777 nodes, 184,812 edges, and 40 different labels.

\subsubsection{Baseline Methods}

We evaluate the performance of Node2Mixture against the following baseline network representation learning methods. For fair comparisons, we set the embedding dimension $d$ as 128 for all methods, other parameters are set as default in original papers if not specified:

- DeepWalk [7]: This approach learns the node embedding as a $d$-dimensional vector by sampling random walks from each node, and applying word2vecbased learning on those walks.

- Node2Vec [2]: This approach learns the node representation as a $d$-dimensional vector by using second-order random walks. The hyper-parameter $p$ and $q$ are data dependent, and are set to achieve the best performances.

- proNE [63]: This approach learns the node representation as a $d$-dimensional vector by using graph spectrum. All hyper-parameters for proNE are set as default to achieve the best performances.

- LINE [3]: This approach is similar to Node2Vec, which obtains $d$-dimensional vector as node's feature representation. The method considers both 1-hop and 2-hop neighborhood to form the loss function. 


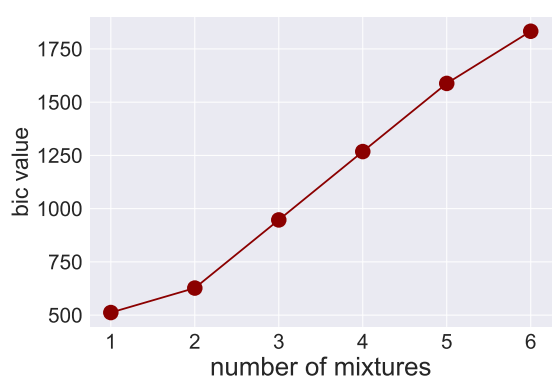

(a)

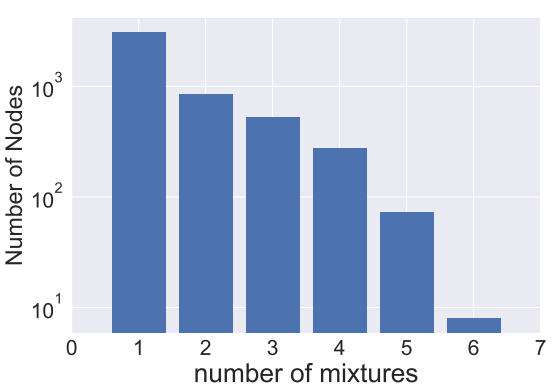

(b)

Fig. 2: (a) BIC score for embedding with different number of mixtures. (b) Distribution of role diversity learned by dynamic mixture embedding learning.

- VERSE [5]: This approach also learns the node representation as a $d$ dimensional vector. The method considered more complicated nodes similarities comparing to Node2Vec or LINE.

- G2G_oh [8]: This approach learns the node representation as a Gaussian Distribution, which obtains $d$-dimensional mean and $d$-dimensional variance. We used the diagonal covariance to match our testing results. Here only the Graph2Gauss one-hot version is tested, because all dataset are plain graph without node content.

\subsection{The Number of Mixture}

\subsubsection{Selecting Number of Components}

As discussed in section 3.5.2, we first determine the optimum setting for the number of mixtures $K$. We first use the Bayesian Information Criterion (BIC) to determine the number of mixtures. Here we use the Wikipedia dataset to demonstrate the results. In Fig. 2a, we treat the BIC as the function as the number of mixtures in GMM for the Wikipedia dataset.

The optimal number of mixture should be the value that minimizes the BIC. According to the figure, we can easily find out that the selection should be the less number mixtures the better. This result matches our recognition on general networks: Most nodes only have one simple role expression, while only a few bridge nodes have the significant embedding diversity.

To further verify our results, we used the dynamic mixture embedding learning (Section 3.5.2) on the Wikipedia dataset, and show the distribution of role diversity in Fig. 2b, i.e., how many nodes have mixture distribution of one, two, three, until seven components. According to the Figure, the majority of nodes have a distribution with only one component, fewer nodes have more than five components in the mixture. This result matches the experimental findings by BIC. 

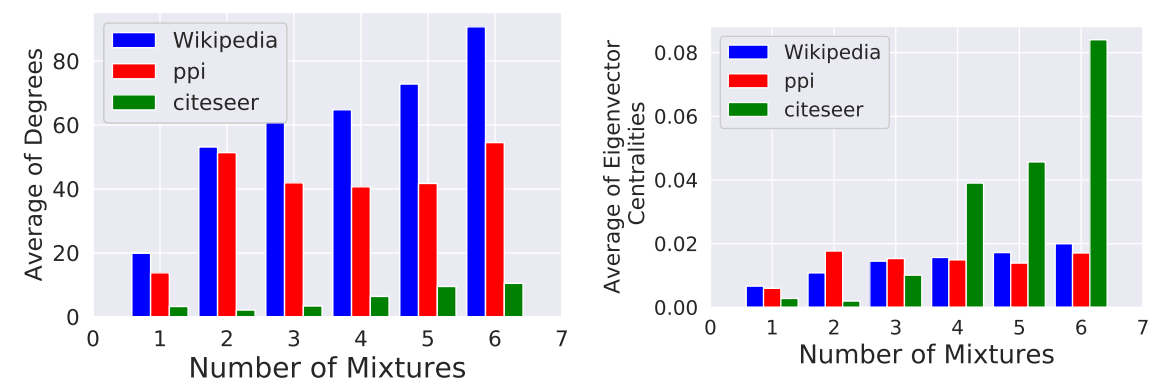

(a) Average degree of nodes with $K$ compo- (b) Average eigenvector centrality of nodes nents in the mixture representation with $K$ components

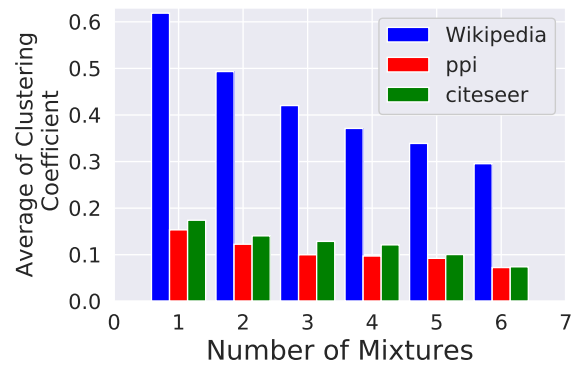

(c) Average cluster coefficient of nodes with $K$ components

Fig. 3: Analysis of network properties w.r.t. the number of components $K$ in the learned mixture embedding by dynamic mixture learning.
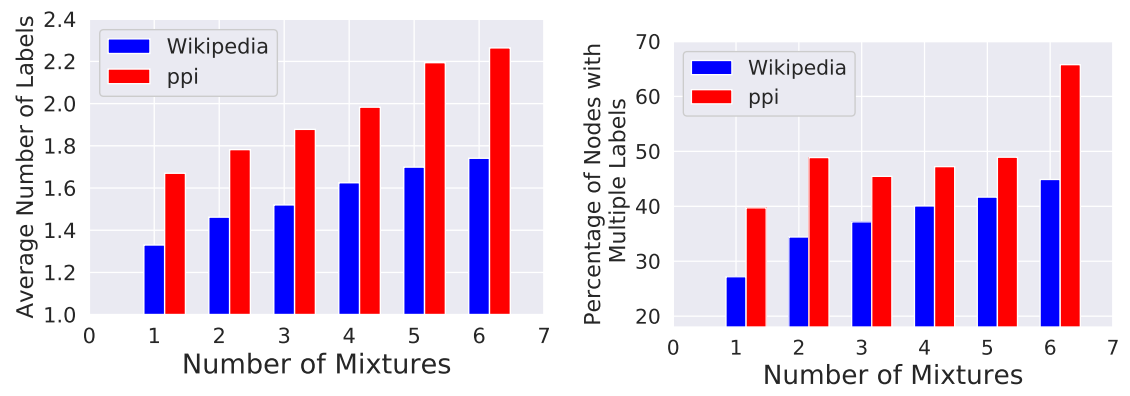

(a) Average number of labels at nodes with

(b) Percentage of nodes with multiple labels different $K$

Fig. 4: The relevance between $K$ and the uncertainty in classification labels.

\subsubsection{The Number of Mixture Components and Network Uncertainty}

Learning the distributional embedding rather than a single point embedding leads us to capture the uncertainty in network better. In the real network, 
such uncertainty always happened for nodes with special functions, such as hub nodes or focal nodes. Here we perform several experiments to evaluate the benefits of our uncertainty modeling.

Same as section 4.2, we adopt the dynamic mixture estimation to obtain the representation of each node. To model the uncertainty, we studied the relationship between several important network topology features and the learned embedding:

- Node Degree: The node degree is the number of edges linked to a node. The larger degree simply indicates that the node is connected to more others.

- Eigenvector centrality: Eigenvector centrality is a measure of the influence of a node in a network. The larger score indicates the higher influential to others the network.

- Clustering coefficient: Clustering coefficient measures whether the nodes are clustered with neighborhood nodes. The higher score means the closer to its neighbors.

Fig. 3 shows that the learned number of mixtures $K$ is correlated with all three network features in three datasets (Wikipedia, PPI and Citeseer). Fig. 3a shows that the learned number of mixtures is large for nodes with higher degrees. In other words, for nodes with high degrees, they tend to be embedded with more Gaussian components, as they are linked with more neighbors and will play various roles. Fig. $3 \mathrm{~b}$ and $3 \mathrm{c}$ show that nodes with higher influences and being less clusterable are more likely represented with more components.

These results indicate that the mixture embedding can reveal the multiple roles nodes are playing. For example, nodes with more Gaussian components tend to have higher eigenvector centrality, which means they are more influential than other nodes in the network. Also, nodes with more Gaussian components are more divergent to its neighbors, as indicated by clustering coefficient. This is because these nodes are possible to be bridge nodes or hub nodes, who have multiple roles in several communities.

For the two multi-label datasets, Wikipedia and PPI, we further evaluate the relevance of $K$ and the uncertainty in classification labels. For a node $n_{i}$, the diversity of its labels reflects its classification uncertainty. In Fig. 4a, nodes with more mixture components tend to have more labels in average. More significantly, Fig. 4b shows that of 70 percent of nodes in PPI with 6 mixture of Gaussian have multiple labels. For nodes embedded by only one Gaussian, there is only less than 40 percent nodes have multiple labels. All the above results show that the mixture Gaussian naturally captures the node characters in networks. For nodes with possible multiple roles, more mixture components are learned.

\subsection{The Embedding of Expected Likelihood Kernel}

The proposed method generates a mixture Gaussian embedding for each vertex. However, the distributional network representation is difficult for using in 
some network tasks, such as node classification and clustering. To better utilize the embedding, we propose to use the expected likelihood kernel distance as the feature representation for downstream tasks. However, using the expected likelihood kernel will result in a $|V|$-dimension distance representation vector for each node $v$, where $|V|$ is the number of nodes in the graph. For a large social network, the $|V|$ can be millions. To reduce the redundant representation information, the Principle Component Analysis (PCA) is used for reducing the dimension of representation and obtain the final representation as the kernel representation mapped to top- $k$ components. Here we study how the selection of $k$ affects the representations.

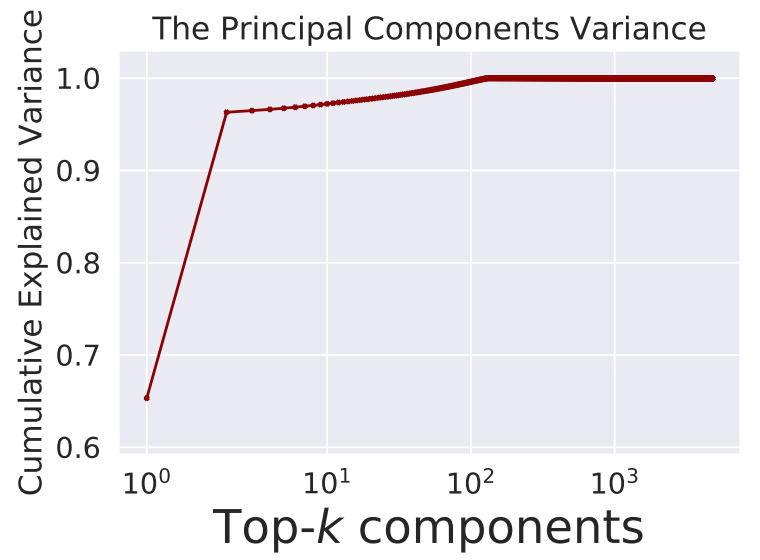

Fig. 5: The cumulative variance for top- $k$ principal components of the expected likelihood kernel matrix from Wikipedia dataset.

We plot the cumulative explained variance of each component for Wikipedia dataset in Fig. 5. In the figure, we visualize the percentage of variance explained by the first $k$ components. Other datasets, such as PPI and Citeseer, share similar behaviors. The first component of the model occupies over 65 percent of all variances. And the explained variance covers about 95 percent among all variances for the top-3 components. And the variance ratio reaches almost 100 percent with about top 100 components. As a result, using PCA to map original kernel representation to $k$ dimension representations, where $k$ should be larger than 10, can keep the critical representation information with much lower dimensions. And the mapped representation will be served as the final representation feature vector.

\subsection{Node Classification}

Node classification is an important and challenging task for network embedding evaluation, as presented in $[2,3,5,7]$. For this task, we used both 


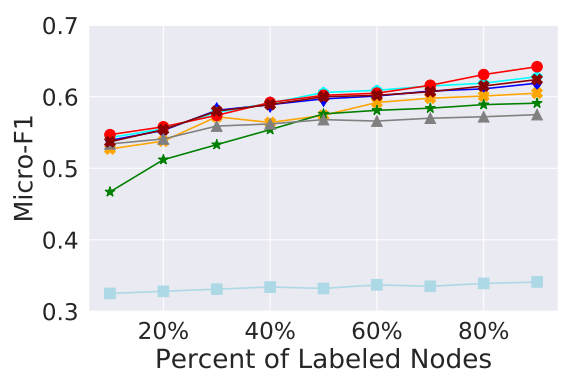

(a) Citeseer by LR

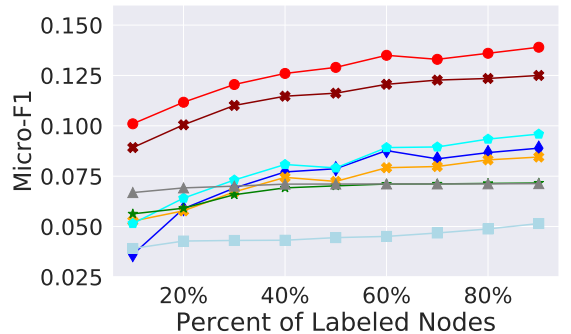

(c) PPI by LR

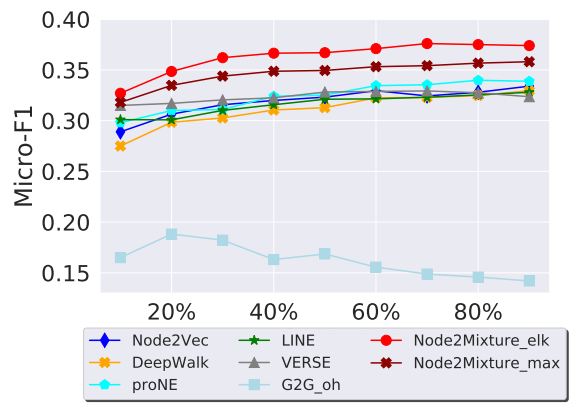

(e) Wiki by LR

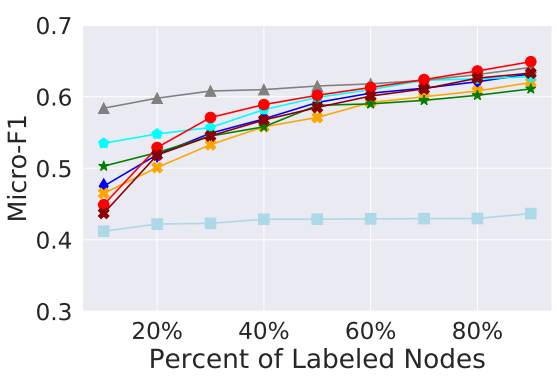

(b) Citeseer by k-nn

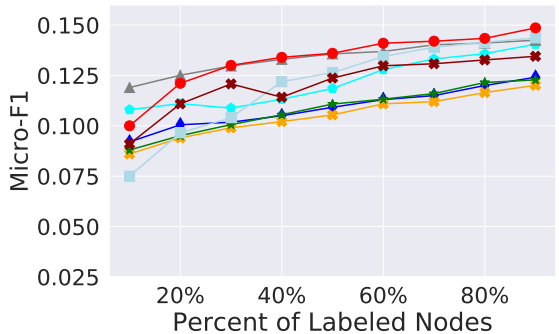

(d) PPI by k-nn

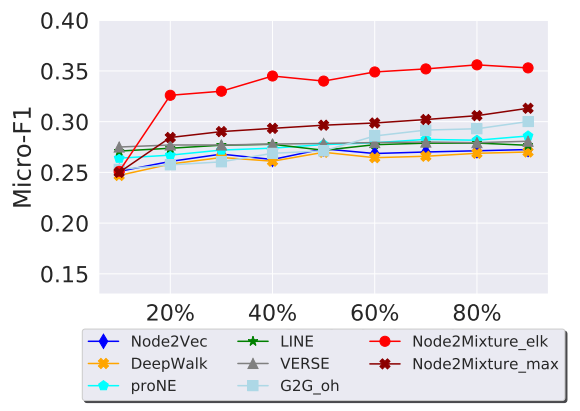

(f) Wiki by k-nn

Fig. 6: Node classification with one-vs-rest logistic regression (LR) classifier and multi-label k-nearest neighbor (k-nn) classifier results for Citeseer, PPI and Wikipedia datasets.

multi-label and multi-class classification dataset for better comparison. For the multi-class node classification experiment, each node is assigned with only one label from the label set. In the multi-label node classification experiment, each node is assigned with one or more labels from the label set. During the training phase, we observe a certain fraction of nodes and their labels, and we predict the labels for the rest of nodes at the testing phase. We evaluate on a multi-class network dataset (Citeseer) and two multi-label network dataset (PPI and Wikipedia) for the node classification. 
Comparing with previous methods, Node2Mixture obtains multiple embedding vectors (both from mean and variance), and thus cannot be directly applied to simple node classification as previous articles did [2,59]. Distributional embedding like [8] only uses the mean vector for node classification, which cannot measure node relevant in the distribution sense. In order to fit the node classification usage, we introduced two variants of Node2Mixture for classification: the expected likelihood kernel as the feature embedding (as introduced in section 4.3) and the mean embedding vector with maximum weight:

- Node2Mixture_elk: This is the proposed method using the expected likelihood kernel as the feature embedding. We use PCA to reduce the representation dimension to $d$, which is the same as other baseline approaches.

- Node2Mixture_max: This is our proposed method using mean embedding vector with maximum weight as the embedding. The parameter settings and embedding dimension $d$ are the same as typical values used for Node2Vec, LINE, Graph2Gauss, etc.

To better evaluate the node classification results, we used two classifiers, One-vs-Rest Logistic Regression and Multi-label k-NN. The former is frequently used for network representation evaluation. However, even using the expected likelihood kernel as feature vector, the mixture distribution cannot avoid using one-vs-rest classifier without losing information, so we also choose the multi-label k-nearest neighbor classifier to predict node labels by similarity measures. Details about classifiers are:

- One-vs-Rest Classifier: The one-vs-rest Logistic Regression classifier is used to fit and make prediction, by taking node embedding as input.

- Multi-Label KNN: The K-nearest neighbor for multi-label classification is used in [60]. In $d$-dimensional embedding space, the cosine similarity is used to find the $k$ nearest neighbors. For distribution based methods, the energy function (either KL-divergence or expected likelihood kernel) is used to determine the $k$ nearest neighbors.

We evaluate the performance by both the Micro-F1 and Macro-F1 percentage measures. However, due to the limitation of space and the similar behavior of two measures, we only report Micro-F1 here. To ensure the reliability, we obtain the experimental results by taking an average of 10 runs, and results are listed in Fig. 6. The x-axis indicates different percentage of labeled nodes.

From the performance of both one-vs-rest logistic regression classifier and k-nearest neighbor multi-label classifier, we can see that our method outperforms the competitors in general. Morever, Node2Mixture_elk performs slightly better than Node2Mixture_max in all three datasets, especially when using the $\mathrm{k}-\mathrm{NN}$ as classifier. This is because Node2Mixture_elk utilizes more information from the distributional representation, while the Node2Mixture_max can only use the major mean vector as the representation. Also, our method especially dominates the performance in two multi-label network dataset, which is a strong sign that using mixture Gaussian embedding is useful. For example, in 
the Wikipedia word co-occurence network, the word "table" can act both as a noun and as a verb. But "table" acts as noun in the majority. Using mixture Gaussian can easily capture the difference between the noun "table" and verb "table" and separate them to two different embeddings, while using a single embedding vector cannot capture the difference and leads to disturbance. Particularly, our method dominates in the Wikipedia dataset when using k-nn, obtains an over 5 percent margin (Fig. 6f).

On the other hand, this leads to the results in multi-class dataset that our method basically performs as good as or slightly better than other methods as Fig $6 \mathrm{a}$ and $6 \mathrm{~b}$. Multi-class dataset contains fewer labels than multi-label cases, and nodes are more likely to present one specific aspect. In this scenario, one of the mixture coefficients will be much bigger than the others, so the uncertainty will not affect the results much. Our mixture embedding will be more like a single Gaussian. Another observation is that our methods have better performance when the training set ratio is larger. This is because when the training ratio is large, mixture Gaussian can overcome the disturbance and the performance rises up quickly as training ratio increases. If the training ratio is small, there will be some disturbance caused by the minor mixture co-efficient distributions.

It is also interesting to notice that Graph2Gauss performs well in both Wikipedia and PPI dataset, with k-nn classier, although not as good as our proposed method. It indicates that distributional distance measure can help bring a tighter relationship for similar nodes. Since distributional representations always contain multiple representation vectors, such as mean and variance vector in Graph2Gauss and our Node2Mixture, selecting any single vector, either mean or variance, as representation will cause representation information loss. For example, with logistic regression classifier on two multi-label datasets (Wiki and PPI), and on multi-class problem of Citeseer, Graph2Gauss is not effective. As a result, distance based classifiers, such as k-nn classifier, is a natural fit for the distributional representation. Distance based classifiers can determine the label by measuring the distance between and existing nodes and predicting nodes, where there is a closed-form to obtain the distance easily, either by ELK (Expected Likelihood Kernel) or KL (Kullback-Leibler) divergence kernel.

In previous papers, the distributional representation cannot directly serve as input for determinitic classifiers, like SVM (Support Vector Machine) or logistic regression, for classification tasks. A simple workaround is to use the mean vector as input [8], but this process ignores the variance vector. In this paper, as mentioned in section 4.3, the Embedding of Expected Likelihood Kernel, expected Likelihood Kernel (ELK) (or any other distance measures) could be used for obtaining the optimum representation vector with dimensional reduction. Using the distance measure can obtain node's single representation vector by measuring distance between pairs of nodes in the graph as a kernel, and the final embedding vector. This process is used to capture the distance features and uses the distance between each node to generate the kernel representation. As mentioned in previous, using ELK kernel can obtain optimum 
Table 1: Link Prediction performance for all datasets.

\begin{tabular}{l|llllll}
\hline \hline \multirow{2}{*}{ Methods } & \multicolumn{2}{|c}{ Citeseer } & \multicolumn{2}{c}{ PPI } & \multicolumn{2}{c}{ Wikipedia } \\
& AUC & AP & AUC & AP & AUC & AP \\
\hline \hline DeepWalk & 0.9579 & 0.9683 & 0.9205 & 0.9287 & 0.8073 & 0.8102 \\
Node2vec & 0.9618 & 0.9718 & 0.9246 & 0.9315 & 0.8134 & 0.8140 \\
proNE & 0.9817 & 0.9848 & 0.9593 & 0.9655 & 0.8875 & 0.8729 \\
LINE & 0.9583 & 0.9618 & 0.9355 & 0.9418 & 0.8043 & 0.8068 \\
VERSE & 0.9892 & 0.9859 & 0.9697 & 0.9622 & $0.9229^{*}$ & $0.9102^{*}$ \\
G2G_oh & 0.9170 & 0.9437 & 0.9093 & 0.9052 & 0.9053 & 0.9092 \\
\hline Node2Mixture_mw & $\mathbf{0 . 9 9 0 5}$ & $\mathbf{0 . 9 9 1 3}$ & $0.9739^{*}$ & $0.9754^{*}$ & 0.8654 & 0.8621 \\
Node2Mixture_elkd & $0.9898^{*}$ & $0.9909^{*}$ & $\mathbf{0 . 9 8 8 3}$ & $\mathbf{0 . 9 8 9 5}$ & $\mathbf{0 . 9 2 5 1}$ & $\mathbf{0 . 9 1 9 2}$ \\
\hline
\end{tabular}

results as well. However, this process is computational costly. The time complexity is $O\left(N^{2}\right)$, where $N$ is the number of nodes in the graph. For large graph with millions of nodes, it is a challenge to compute kernel efficiently.

Distributional representations can be used for downstream tasks, like node classification or link prediction. However, a carefully selected classifier can cooperate with distributional representation and leads to a better performance in adaptive multi-label experiments. Using distance based classifiers or obtaining the distance kernel as representation are both the proper approaches.

\subsection{Link Prediction}

Link prediction is another commonly used network representation evaluation task. We evaluate the performance of the methods by hiding a set of edges/nonedges from the original path and training on the resulting graph. Following the experiment settings in [8], we create a link test set with $10 \%$ randomly selected edges set and equal number of randomly selected non-edges. We used all three datasets tested in the node classification experiments as in section 4.4.

We report the area under ROC curve (AUC) and the Average Precision (AP) scores for each method as by convention [8]. To score the baseline methods, we use the exact same approach as in the respective original methods (e.g. dot product of the vector embeddings or KL-divergence in Graph2Gauss).

As the KL-divergence can only be approximate between mixture of Gaussians, we used two different similarity distance measures to score our method:

- Node2Mixture_elkd: This is the proposed method using Expected Likelihood Kernel Distance for similarity measure as it is used as the energy function. ELK Distance measures the similarity between two distributions $p_{i}$ and $p_{c}$ by:

$$
d\left(p_{i}, p_{c}\right)=\int p_{i}(x) p_{c}(x) d x
$$

- Node2Mixture_mw: This is the proposed method using mean embedding vector with maximum weight as the node embedding and measures the similarity of nodes by the dot product of the vector embeddings. 
The performances on the link prediction task are listed in Table 1. We can see that both distance measurements in our method outperform the competitors across most datasets. This indicates that the learned mixture embeddings are especially useful to capture information between pairs of nodes. The ELK measure can obtain almost the best among all datasets, while having very close performance to the max weight measurement on Citeseer dataset. We attribute this character to the extra information captured by the variance in ELK measure.

\subsection{Cases Study}

In the previous experiments, we evaluate how Node2Mixture can well capture the uncertainty of networks. Here we want to empirically evaluate two cases, Les Misérable network and Karate network, due to their rich information available about nodes and edges. We show the discovery of uncertainties in Les Misérable network by analyzing the representations of nodes with different functions.

\subsubsection{Les Misérable Network}

We use a fiction network, where nodes correspond to characters in the novel Les Misérables [61]. Edges connect co-appearing characters. The network has 77 nodes and 254 edges. We set embedding space to 64 dimensions for representation each node.

Fig. 7 presents the visualization results for Les Misérable using Node2Mixture and Node2Vec [2]. The size of a node indicates its degree. Different colors in Fig. $7 \mathrm{a}$ is to indicate the different number of mixtures learned. Blue nodes have three components, yellow nodes have two components, while red ones have only one component. Node2Vec's embedding results are obtained with parameter $p=1, q=2$. In Fig. $7 \mathrm{~b}$, nodes in the same color mean the same structural function, as evaluated in [2].

Due to the different learning targets, here we do not intend to compare the effectiveness of node characterization. Instead, Node2Vec's embedding can be a reference for Node2Mixture. Comparing two figures in Fig. 7, we can find out that nodes learned with the same number of components are more likely to be structurally similar. For instance, Node2Mixture embeds almost all the "blue" nodes in Fig. 7b to single Gaussian distribution. Because these nodes are characters at the periphery and have limited interactions in the novel. Node2Mixture embeds many bridge nodes to a mixture of three Gaussian distributions (colored by blue in Fig. 7a). These nodes represent characters that act at several sub-plots of the novel simultaneously. For instance, the upper left and upper right blue nodes in Fig 7a represents "Fantine" and "Myriel", respectively. Both characters not only interact with the hub node "Valjean" in the main story, but also have their own subplots. Such as "Fantine" has her own story with students "Tholomyes" and his friends, and "Myriel" interacts 


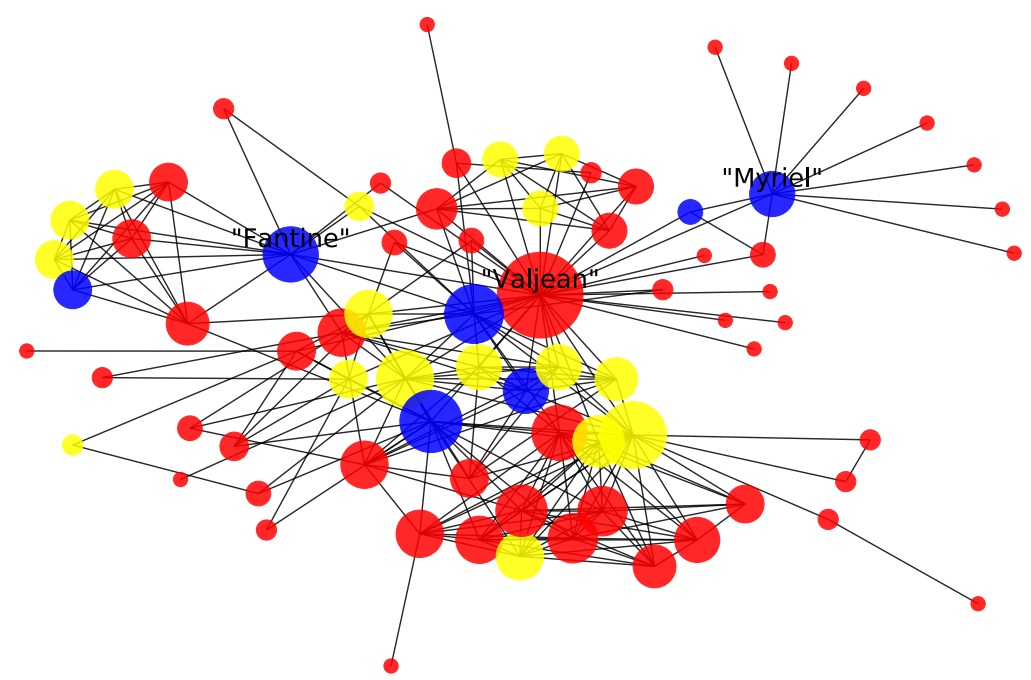

(a) Node2Mixture embedding visualization in Les Misérables. Blue (yellow, red) nodes have three (two, one) components.

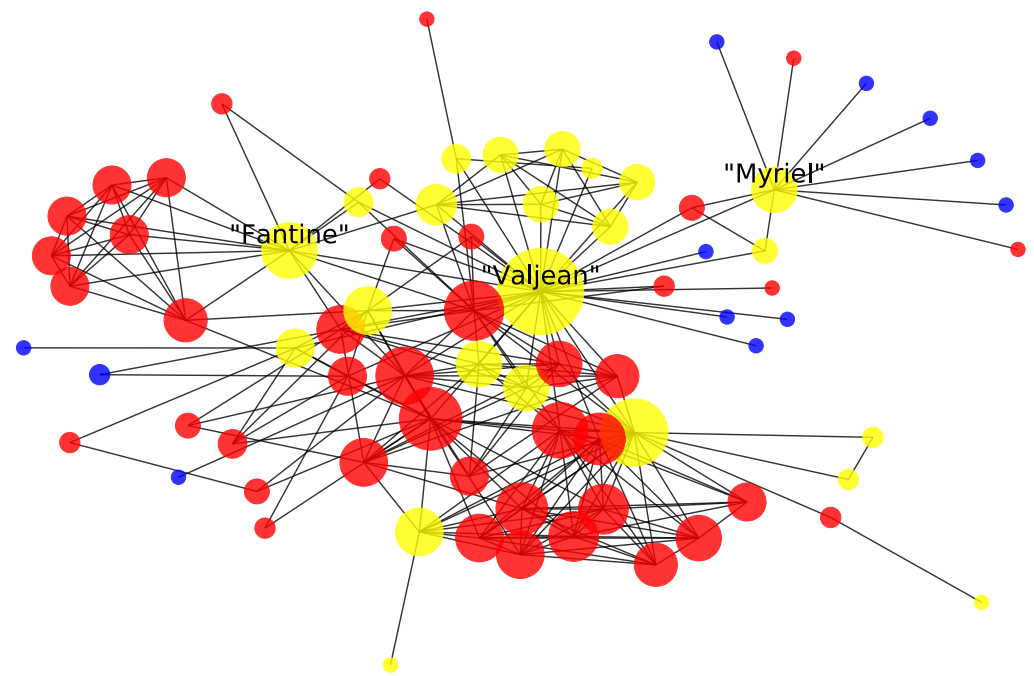

(b) Node2Vec embedding visualization in Les Misérables. Same color means the same structural function.

Fig. 7: Empirical evaluation of Les Misérables characters co-appearance network. The upper figure is the generated by Node2Mixture. The bottom is the structural embedding by Node2Vec.

with several independent characters, such as meeting with Napoleon. Important characters such as "Cosette", "Marius" and "Javert" are also embedded 
with multiple Gaussian components. These characters all played in multiple independent storylines.

\subsubsection{Karate Network}

Karate club network is another graph we would love to illustrate here. Karate club network is a social network describing about the university karate club. The network captured 34 members of a karate club, documenting links between pairs of members who interacted outside the club.

In the experiment, different from the Les Misérable network, Karate network has much less vertex and simpler relations. So we can visualize the representations in a 2-dimension space. We first plot the Karate network with spring layout in Fig. 8a. In the figure, we colored the node blue, red and green. The blue and red nodes stand for two separate clusters labeled by data sources. The green nodes stands for bridge nodes that happened to be in the middle of two clusters, more specifically, have more than 1 connection in both clusters. we visualize the node representation from Karate club network in Fig. 8b. Each node is firstly embed to a 128 dimension vector mean and 128-dim diagonal variance. Then we use the t-SNE to reduce both mean and variance to 2 dimensional, and plot in Fig. 8b. We used the same color to indicate nodes with same labels.

As presented in Fig. 8b, we can easily find a separation plane between different classes (blue and red). Moreover, we embed the bridge nodes with multiple mixtures to green color in the figure. For these hub nodes, we use (0) to indicate the major Gaussian and (1) to the minor Gaussian. These nodes all act as bridges between different classes in the graph. Using mixture Gaussian embedding is thus meaningful. For example, in the original karate graph, node 8 connected to both node 32 and 33 in the red cluster, as well as 13 and 0 in the blue clusters. In the visualization, node 8 has two different Gaussian means, the major Gaussian is closer to the "red" class, and the minor Gaussian is in the red node cluster. This is due to the fact that node 8 has almost the same number of neighbors in class "blue" and "red". This phenomenon also happens for node 2 and 31. The mixture embedding will learn two distribution centers at different group, and the Gaussian with a larger mixture coefficient always belongs to the correct group. Further, node 30 connects to both node 32 and 33 in the red cluster, while only connects to node 1 in the blue cluster. The representation reflects the connection well that node 30 has a major Gaussian distribution within the blue cluster, while has another mean surrounded in the separation line between the red and blue clusters. In general, Node2Mixture can well present the meaningful embeddings of the network. Especially for the bridge nodes, Node2Mixture can capture the multiformity of their features and generate explainable mixture embedding. 


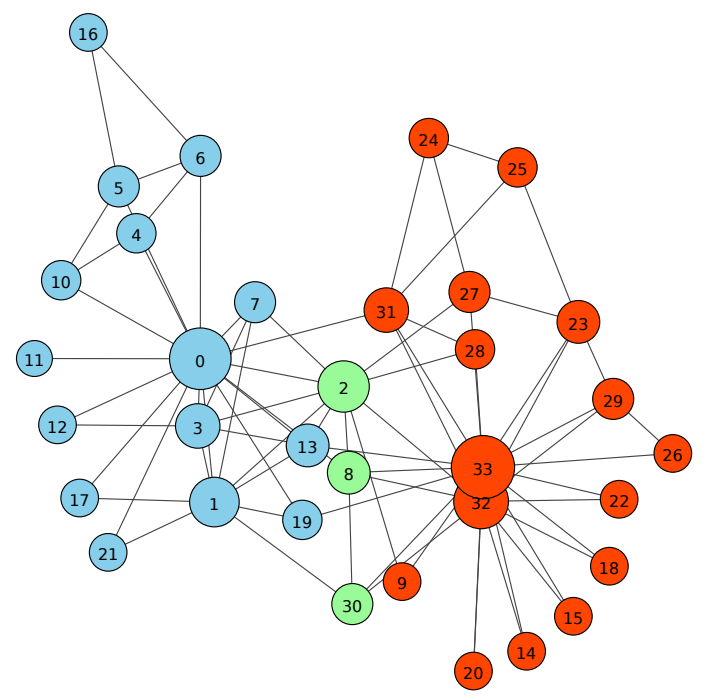

(a) The Karate Network.

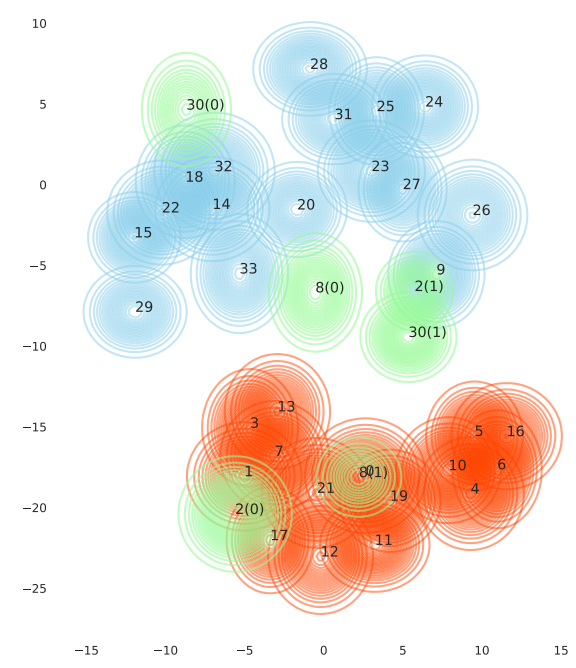

(b) Node2Mixture embedding of Karate Network.

Fig. 8: Empirical evaluation of the Karate Network. The upper figure is the original Karate Network, the bottom is the visualization of Node2Mixture embedding. 


\section{Conclusion}

We propose a novel network embedding method that represents each node as a mixture of Gaussian distributions, and therefore be able to capture the multiple possible roles each node is playing. The resulted mixture distribution representation enables the comparison of node pairs in expected likelihood kernel which is beneficial to the downstream graph applications. Experiments show the mixture representations learned by Node2Mixture can outperform other methods in node classification and link prediction. Analyzing the graph features gives the evidence that embedding node to Gaussian mixture can better model the uncertainty of a node.

Representing node by mixture embedding is a promising solution for capturing the uncertainty in graph. In our future work, we consider to upgrade our model for learning more comprehensive representations from multi-source network. Besides working on plain networks, network embedding can also fuse node attributes or edge information. A straight forward extension of our method is to build inductive learning models for attributed network. For example, inferencing the nodes' mixture of Gaussian representations by learning a mapping function that takes input of both node attributes and graph structures. Further, by incorporating network attributes, both attributes and nodes can be embedded in the same representation space, a.k.a. co-embedding problem $[69,70]$. As a result, both attributes and nodes can be represented as mixture of distributions, and used for downstream tasks for node classification, link predictions and feature inference

\section{Acknowledgement}

This work was partially supported and funded by King Abdullah University of Science and Technology (KAUST), under award number FCC/1/1976-19-01, and NSFC No 61828302, the National Key Research and Development Program of China (2017YFB1002000), Science Technology and Innovation Commission of Shenzhen Municipality (JCYJ20180307123659504), and the State Key Laboratory of Software Development Environment in Beihang University.

\section{References}

1. L. Tang and H. Liu, "Leveraging social media networks for classification," Data Mining and Knowledge Discovery, vol. 23, no. 3, pp. 447-478, 2011.

2. A. Grover and J. Leskovec, "node2vec: Scalable feature learning for networks," in SIGKDD. ACM, 2016, pp. 855-864.

3. J. Tang, M. Qu, M. Wang, M. Zhang, J. Yan, and Q. Mei, "LINE: Large-scale Information Network Embedding," $W W W$, pp. 1067-1077, 2015. [Online]. Available: http://arxiv.org/abs/1503.03578

4. P. Cui, X. Wang, J. Pei, and W. Zhu, "A survey on network embedding," IEEE Transactions on Knowledge and Data Engineering, 2018. 
5. A. Tsitsulin, D. Mottin, P. Karras, and E. Müller, "Verse: Versatile graph embeddings from similarity measures," in Proceedings of the 2018 World Wide Web Conference, ser. WWW, 2018, pp. 539-548.

6. Xiaofang Zhou, "Destination-aware Task Assignment in Spatial Crowdsourcing: A Worker Decomposition Approach." in IEEE Trans. Knowl. Data Eng., DOI: 10.1109/TKDE.2019.2922604, 2019

7. B. Perozzi, R. Al-Rfou, and S. Skiena, "Deepwalk: Online learning of social representations," in SIGKDD. ACM, 2014, pp. 701-710.

8. A. Bojchevski and S. Günnemann, "Deep gaussian embedding of attributed graphs: Unsupervised inductive learning via ranking," ICLR, 2018.

9. T. Jebara, R. I. Kondor, and A. Howard, "Probability product kernels," JMLR, vol. 5, pp. 819-844, 2004.

10. W. L. Hamilton, R. Ying, and J. Leskovec, "Representation learning on graphs: Methods and applications," arXiv preprint arXiv:1709.05584, 2017.

11. H. Cai, V. W. Zheng, and K. Chang, "A comprehensive survey of graph embedding: problems, techniques and applications," TKDE, 2018.

12. G. Sun, X. Zhang. "A Novel Framework for Node/Edge Attributed Graph Embedding", in $P A K D D, 2019$

13. U. Akujuobi, H. Yufei, Q. Zhang, X. Zhang. "Collaborative Graph Walk for Semisupervised Multi-Label Node Classification", in ICDM, 2019.

14. X. Chen, G. Yu, J. Wang, C. Domeniconi, Z. Li, X. Zhang. "ActiveHNE: Active Heterogeneous Network Embedding". In IJCAI, 2019.

15. K. Zheng, Y. Zhao, D. Lian, B. Zheng, .G. Liu, X. Zhou, "Reference-based Framework for Spatio-temporal Trajectory Compression and Query Processing." in TKDE, 2019

16. M. Belkin and P. Niyogi, "Laplacian eigenmaps and spectral techniques for embedding and clustering," in NIPS, 2002, pp. 585-591.

17. S. T. Roweis and L. K. Saul, "Nonlinear dimensionality reduction by locally linear embedding," science, vol. 290, no. 5500, pp. 2323-2326, 2000.

18. Y. Bengio, A. Courville, and P. Vincent, "Representation learning: A review and new perspectives," IEEE TPAMI, vol. 35, no. 8, pp. 1798-1828, 2013.

19. D. Lian, K. Zheng, Y. Ge, L. Cao, E. Chen, X. Xie: "GeoMF++: Scalable Location Recommendation via Joint Geographical Modeling and Matrix Factorization." in $A C M$ Trans. Inf. Syst. 36(3): 33:1-33:29, 2018

20. S. Cao, W. Lu, and Q. Xu, "Deep neural networks for learning graph representations." in $A A A I, 2016$, pp. 1145-1152.

21. D. Zhang, J. Yin, X. Zhu, and C. Zhang, "User profile preserving social network embedding," in Proceedings of the 26th International Joint Conference on Artificial Intelligence. AAAI Press, 2017, pp. 3378-3384.

22. A. Ahmed, N. Shervashidze, S. Narayanamurthy, V. Josifovski, and A. J. Smola, "Distributed large-scale natural graph factorization," in $W W W$. ACM, 2013, pp. 37-48.

23. L. F. Ribeiro, P. H. Saverese, and D. R. Figueiredo, "struc2vec: Learning node representations from structural identity," in Proceedings of the 23rd ACM SIGKDD International Conference on Knowledge Discovery and Data Mining. ACM, 2017, pp. 385-394.

24. M. Qu, J. Tang, J. Shang, X. Ren, M. Zhang, and J. Han, "An attention-based collaboration framework for multi-view network representation learning," in Proceedings of the 2017 ACM on Conference on Information and Knowledge Management. ACM, 2017, pp. $1767-1776$.

25. L. Li, K. Zheng, S. Wang, X. Zhou, "Go Slow to Go Fast: Minimal On-Road Time Route Scheduling with Parking Facilities Using Historical Trajectory" in VLDB Journal 27(3): 321-345, 2018

26. Y. Ma, Z. Ren, Z. Jiang, J. Tang, and D. Yin, "Multi-dimensional network embedding with hierarchical structure," WSDM, 2018.

27. B. Perozzi, V. Kulkarni, H. Chen, and S. Skiena, "Don't walk, skip!: Online learning of multi-scale network embeddings," in Proceedings of the 2017 IEEE/ACM International Conference on Advances in Social Networks Analysis and Mining 2017. ACM, 2017, pp. 258-265.

28. J. Tang, M. Qu, and Q. Mei, "Identity-sensitive word embedding through heterogeneous networks," arXiv preprint arXiv:1611.09878, 2016. 
29. S. He, K. Liu, G. Ji, and J. Zhao, "Learning to represent knowledge graphs with gaussian embedding," in Proceedings of the 24th ACM International on Conference on Information and Knowledge Management. ACM, 2015, pp. 623-632.

30. L. Dos Santos, B. Piwowarski, and P. Gallinari, "Multilabel classification on heterogeneous graphs with gaussian embeddings," in Joint European Conference on Machine Learning and Knowledge Discovery in Databases. Springer, 2016, pp. 606-622.

31. D. Reynolds, "Gaussian mixture models," Encyclopedia of biometrics, pp. 827-832, 2015.

32. M. Balafar, "Gaussian mixture model based segmentation methods for brain mri images," Artificial Intelligence Review, vol. 41, no. 3, pp. 429-439, 2014.

33. T. Higuchi, N. Ito, S. Araki, T. Yoshioka, M. Delcroix, and T. Nakatani, "Online mvdr beamformer based on complex gaussian mixture model with spatial prior for noise robust asr," IEEE/ACM Transactions on Audio, Speech, and Language Processing, vol. 25, no. 4, pp. 780-793, 2017.

34. G. Liu, K. Zheng, A. Liu, Z. Li, Y. Wang, X. Zhou "MCS-GPM: Multi-Constrained Simulation Based Graph Pattern Matching in Contextual Social Graphs." in TKDE 30(6): 1050-1064, 2018

35. C. Zhang and P. Woodland, "Joint optimisation of tandem systems using gaussian mixture density neural network discriminative sequence training," in ICASSP. IEEE, 2017, pp. 5015-5019.

36. C. Bouveyron and C. Brunet-Saumard, "Model-based clustering of high-dimensional data: A review," Computational Statistics \&S Data Analysis, vol. 71, pp. 52-78, 2014.

37. X. Yang, K. Huang, J. Y. Goulermas, and R. Zhang, "Joint learning of unsupervised dimensionality reduction and gaussian mixture model," Neural Processing Letters, vol. 45, pp. 791-806, 2017.

38. M. Paulik, "Lattice-based training of bottleneck feature extraction neural networks." in Interspeech, 2013, pp. 89-93.

39. X. Chen, X. Qiu, J. Jiang, and X. Huang, "Gaussian mixture embeddings for multiple word prototypes," CoRR, vol. abs/1511.06246, 2015.

40. B. Athiwaratkun and A. G. Wilson, "Multimodal word distributions," in Conference of the Association for Computational Linguistics (ACL), 2017.

41. Y. LeCun, S. Chopra, R. Hadsell, M. Ranzato, and F. Huang, "A tutorial on energybased learning," Predicting structured data, vol. 1, no. 0, 2006.

42. J. Mueller and A. Thyagarajan, "Siamese recurrent architectures for learning sentence similarity." in AAAI, 2016, pp. 2786-2792.

43. P. Neculoiu, M. Versteegh, and M. Rotaru, "Learning text similarity with siamese recurrent networks," in Proceedings of the 1st Workshop on Representation Learning for NLP, 2016, pp. 148-157.

44. R. Tao, E. Gavves, and A. W. Smeulders, "Siamese instance search for tracking," in CVPR. IEEE, 2016, pp. 1420-1429.

45. B. Zheng, H. Su, W. Hua, K. Zheng, X. Zhou, G. Li "Efficient Clue-Based Route Search on Road Networks" in TKDE 29(9): 1846-1859, 2017

46. Y.-l. Boureau, Y. L. Cun et al., "Sparse feature learning for deep belief networks," in NIPS, 2008, pp. 1185-1192.

47. J. R. Hershey and P. A. Olsen, "Approximating the kullback leibler divergence between gaussian mixture models," ICASSP, vol. 4, pp. IV-317-IV-320, 2007.

48. T. Jebara and R. Kondor, "Bhattacharyya and expected likelihood kernels," in Learning theory and kernel machines. Springer, 2003, pp. 57-71.

49. Z. Yang, W. Cohen, and R. Salakhutdinov, "Revisiting Semi-Supervised Learning with Graph Embeddings," ICML, vol. 48, 2016. [Online]. Available: http://arxiv.org/abs/ 1603.08861

50. R. Hadsell, S. Chopra, and Y. LeCun, "Dimensionality reduction by learning an invariant mapping," in $C V P R$, vol. 2. IEEE, 2006, pp. 1735-1742.

51. L. Vilnis and A. Mccallum, "Word representations via gaussian embedding," ICLR, pp. $1-12,2015$.

52. O. Levy, Y. Goldberg, and I. Dagan, "Improving distributional similarity with lessons learned from word embeddings," Transactions of the Association for Computational Linguistics, vol. 3, pp. 211-225, 2015. 
53. J.-L. Durrieu, J.-P. Thiran, and F. Kelly, "Lower and upper bounds for approximation of the kullback-leibler divergence between gaussian mixture models," ICASSP, pp. 48334836, 2012.

54. X. Gao and R. J. Carroll, "Data integration with high dimensionality," Biometrika, vol. 104, no. 2, pp. 251-272, 2017.

55. C. Yang, Z. Liu, D. Zhao, M. Sun, and E. Y. Chang, "Network representation learning with rich text information," IJCAI, vol. 2015-Janua, pp. 2111-2117, 2015.

56. B.-J. Breitkreutz, C. Stark, T. Reguly, L. Boucher, A. Breitkreutz, M. Livstone, R. Oughtred, D. H. Lackner, J. Bähler, V. Wood et al., "The biogrid interaction database," Nucleic acids research, vol. 36, no. suppl_1, pp. D637-D640, 2008.

57. K. Zheng, Y. Zheng, N. J. Yuan, S. Shang, X. Zhou "Online Discovery of Gathering Patterns over Trajectories" in IEEE Trans. Knowl. Data Eng. 26(8): 1974-1988, 2014

58. M. Mahoney, "Large text compression benchmark," URL: http://www. mattmahoney. net/text/text. html, 2011.

59. W. L. Hamilton, R. Ying, and J. Leskovec, "Inductive representation learning on large graphs," in NIPS, 2017.

60. M.-L. Zhang and Z.-H. Zhou, "Ml-knn: A lazy learning approach to multi-label learning," Pattern Recognition, vol. 40, no. 7, pp. 2038-2048, 2007.

61. D. E. Knuth, The Stanford GraphBase: a platform for combinatorial computing. Addison-Wesley Reading, 1993, vol. 37.

62. Epasto, A., Perozzi, B., "Is a Single Embedding Enough? Learning Node Representations that Capture Multiple Social Contexts" In The Web Conference, 2019.

63. J. Zhang and Y. Dong and Y. Wang and J. Tang and M. Ding, "ProNE: Fast and Scalable Network Representation Learning" In IJCAI, 2019

64. X. Liu and T. Murata and K. Kim and C. Kotarasu and C. Zhuang, "A General View for Network Embedding as Matrix Factorization" in WSDM, 2019

65. R. Yang and J. Shi and X. Xiao and S. S. Bhowmick and Y.J. Yang "Homogeneous Network Embedding for Massive Graphs via Personalized PageRank", in ArXiv, 2019

66. J. Qiu and Y. Dong and H. Ma and J. Li and K. Wang and J. Tang "Network Embedding as Matrix Factorization: Unifying DeepWalk, LINE, PTE, and node2vec", in WSDM, 2018

67. D. Zhu and P. Cui and D. Wang and W. Zhu "Deep Variational Network Embedding in Wasserstein Space", in KDD, 2018

68. J. Jiang, D. Yang, Y. Xiao and C. Shen. "Convolutional Gaussian Embeddings for Personalized Recommendation with Uncertainty", in IJCAI, 2019.

69. S. Liang, X. Zhang, Z. Ren, E. Kanoulas. "Dynamic Embeddings for User Profiling in Twitter", in $K D D, 2018$

70. Z. Meng, S. Liang, H. Bao, X. Zhang. "Co-Embedding Attributed Networks", in WSDM, 2019 . 